\title{
On the operation of silicon photomultipliers at temperatures of $1-4$ kelvin
}

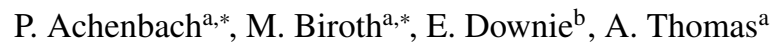 \\ ${ }^{a}$ Institut für Kernphysik, Johannes Gutenberg-Universität, Mainz, Germany \\ ${ }^{b}$ Physics Department, George Washington University, Washington DC, USA
}

\begin{abstract}
SiPM operation at cryogenic temperatures fails for many common devices. A particular type from Zecotek with deep channels in the silicon substrate instead of quenching resistors was tested at liquid helium temperature. Two similar types were thoroughly characterized from room temperature down to liquid nitrogen temperature by illuminating them with low light levels. At cryogenic temperatures the SiPMs show an unchanged rise-time and a fast recovery time, practically no after-pulses, and exhibit no increased cross-talk probability. Charge collection spectra were measured to extract the pixel gain and its variation, both comparable to room temperature at the same over-voltage. The quenching resistance was decreased at cryogenic temperature. It was found possible to use the characterized devices at temperatures of $1-4 \mathrm{~K}$ for the read-out of a target at the Mainz Microtron in Germany.
\end{abstract}

Keywords: Silicon photomultiplier (SiPM), micro-pixel avalanche photo diodes (MAPD), cryogenic temperatures, charge collection spectra

PACS: 85.60.Gz, 85.60.Ha, 07.20.Mc

\section{Introduction}

Silicon PhotoMultipliers (SiPMs) are being considered as possible photodetectors operating at low temperatures for direct dark matter searches, neutrino detectors, telescopes in space, or at high energy beams.

For photonuclear experiments at the Mainz Microtron MAMI, Germany, we are developing a charged particle detector immersed in the mixed ${ }^{3} \mathrm{He} /{ }^{4} \mathrm{He}$ liquid helium of a dilution refrigerator. In the most extreme position the SiPM devices have to be operated at temperatures between 1 and $4 \mathrm{~K}$ [1].

When operating SiPMs or other solid-state devices for photon detection at cryogenic temperatures not only the electronic properties of silicon can change but also fundamental device properties. At cryogenic temperature the charge carrier freezeout depletes the device. Some SiPM types, e.g. Hamamatsu SiPM of type S10362-11-100P, fail to operate because of their lost ability to quench pixel discharges, others exhibit a nontolerable increase in after-pulsing probability [2]. In Ref. [3] an example is shown where the recovery times of Hamamatsu types MPPC S10362-11-050U-3 and MPPC S10931-33050P-63813 were increasing from $\sim 35 \mathrm{~ns}$ to $\sim 230 \mathrm{~ns}$ for the $1 \times 1 \mathrm{~mm}^{2}$ detector and from $\sim 45$ to $\sim 160$ for the $3 \times 3$ detector when lowering the temperature from $328 \mathrm{~K}$ to $98 \mathrm{~K}$. Such a characteristic, explained by the increase in the quenching resistance of polycrystalline silicon resistors, is not tolerable for fast ${ }_{30}$ photon counting experiments.

Initial tests have shown that one particular type of SiPM, ${ }_{11}$ MAPD-3N by Zecotek [4] with deep channels in the silicon 32

*Corresponding authors: Tel.: +49 61313925777 , Fax: +4961313922964. ${ }^{34}$ Email addresses: patrick@kph.uni-mainz.de (P. Achenbach), biroth@kph.uni-mainz.de (M. Biroth)

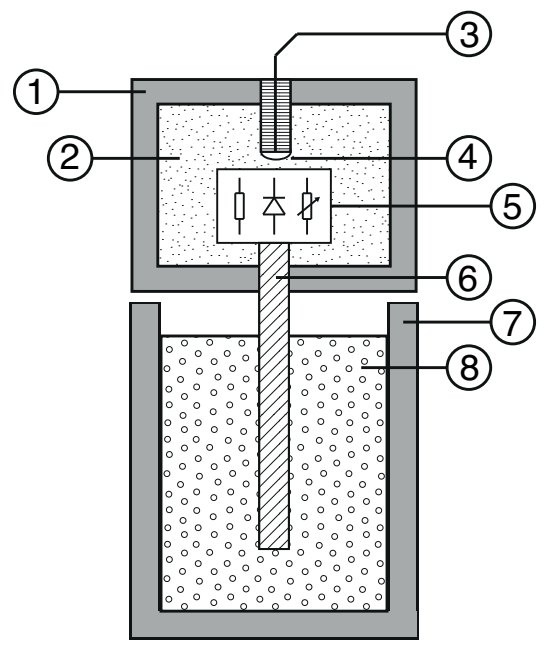

Figure 1: SiPM cryogenic test setup: (1) light and vacuum tight housing, (2) low pressure $p<10^{-6}$ mbar to minimize heat exchange, (3) light guide from LED pulser, (4) focusing screen, (5) cold-head with 4 SiPMs, 2 temperature probes and 2 heaters, (6) cold-finger thermally coupled to cold-head, (7) liquid nitrogen dewar, (8) liquid nitrogen surrounding cold-finger.

substrate instead of quenching resistors, can be successfully operated immersed in liquid helium at a temperature of $4 \mathrm{~K}$ [2].

We have continued our tests with the type MAPD-3NK0, with an active area of $3.7 \times 3.7 \mathrm{~mm}^{2}$ and a pixel density of 15000 pixels $/ \mathrm{mm}^{2}$, as well as with type MAPD-3N1P, with an active area of $3.0 \times 3.0 \mathrm{~mm}^{2}$ and a pixel density of 10000 pixels $/ \mathrm{mm}^{2}$. All SiPMs were without epoxy layer which is problematic for cryogenic operation. 


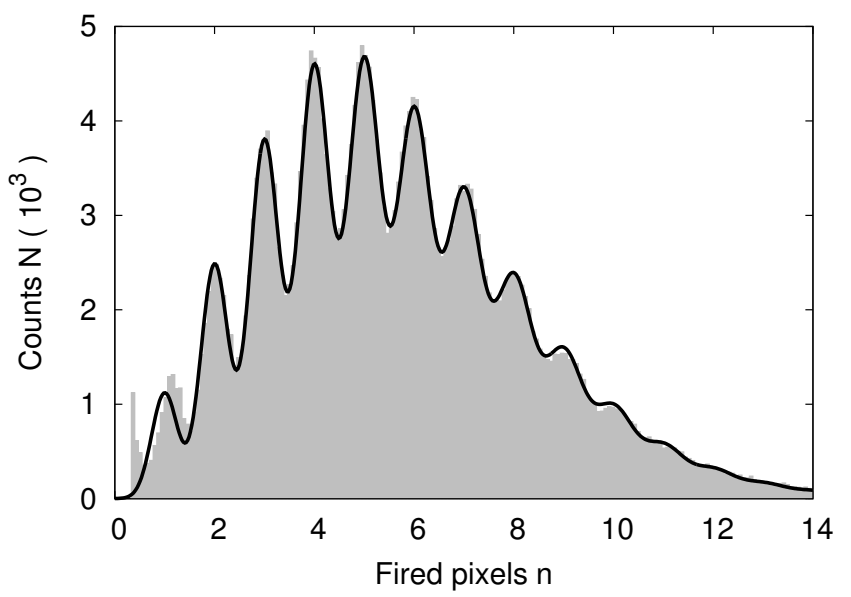

Figure 2: Charge spectrum of Zecotek SiPM type MAPD-3NK0 at an average intensity of 4.9 photons and a temperature of $100 \mathrm{~K}$. A fit was performed to ${ }^{53}$ extract SiPM properties and the light intensity. The single-pixel gain was de- 54 termined to be $6.6 \times 10^{4}$ electron charges. Because of the low pedestal charge 55 the position of the first pixel peak is de-localized.

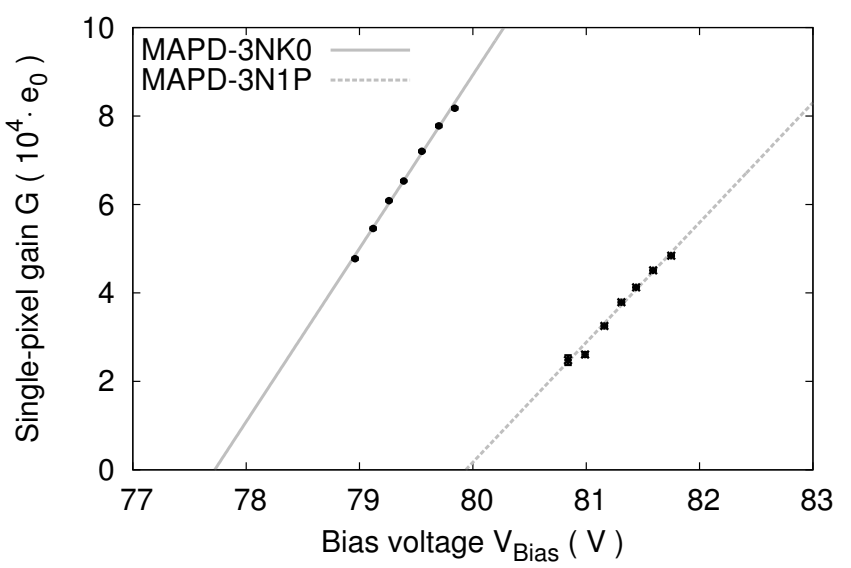

Figure 3: Single-pixel gain as a function of bias voltage of two types of Zecotek 73 SiPMs at a temperature of $100 \mathrm{~K}$. The values were obtained by fitting of SiPM $_{74}$ charge spectra at different bias voltages.

Table 1: Fit parameters of the charge spectra of two types of Zecotek SiPMs 77 measured at $100 \mathrm{~K}$.

\begin{tabular}{lcc}
\hline measured at $100 \mathrm{~K}$. & MAPD-3NK0 & MAPD-3N1P \\
\hline Parameters & 79.39 & 81.31 \\
Over-voltage $(\mathrm{V})$ & $1.66 \pm 0.14$ & $1.55 \pm 0.07$ \\
\hline Pixel gain $G\left(10^{4} \times e_{0}\right)$ & $6.60 \pm 0.01$ & $3.79 \pm 0.02$ \\
Pixel variation $(\% G)$ & $10.3 \pm 0.3$ & $13.1 \pm 1.1$ \\
Crosstalk $(\%)$ & $11.9 \pm 0.3$ & $8.2 \pm 0.5$ \\
\hline Average no. of photons & $4.88 \pm 0.02$ & $2.89 \pm 0.02$ \\
\hline \hline
\end{tabular}

\section{Experimental set-up and measurements}

The devices were mounted inside a vacuum chamber, anchored to a liquid nitrogen cold-head. A shielding provided further thermal insulation. The temperature of the set-up was stabilized with a feedback mechanism, a commercially available proportional-integral-derivative (PID) temperature controller and heating resistors, to allow long-term operation from room temperature down to liquid nitrogen temperature. Signals from a LED pulser were brought via a light guide to the SiPM site. The layout of the cryogenic test set-up is shown in Fig. 1. A fast signal acquisition system was used to acquire charge collection spectra with single-pixel resolution. The acquisition has been triggered by timing signals given by the LED system.

The dark count rate dropped exponentionally with temperature. At cryogenic temperatures the SiPM signals show an unchanged rise-time of $10 \mathrm{~ns}$ and a fast recovery-time of $25.5 \pm$ $0.8 \mathrm{~ns}$ as compared to $35.0 \pm 2.5 \mathrm{~ns}$ at $300 \mathrm{~K}$. Practically no after-pulses and no increased cross-talk probability were observed. The charge spectrum of SiPM type MAPD-3NK0 at an average intensity of 4.9 photons and a temperature of $100 \mathrm{~K}$ is given in Fig. 2. The charge spectra were fitted with the model presented in Ref. [2]. The fit delivered SiPM properties like single-pixel gain $G$, crosstalk probability, single-pixel variation relative to gain, and the average light intensity, see Table 1. Most SiPM properties were found to be comparable to room temperature at the same over-voltage $\Delta V=V_{\text {bias }}-V_{B D}$. The $\mu$ cell capacitance $C_{\mu c e l l}$ was determined from the gain equation $G=Q / e_{0}=C_{\mu c e l l} \Delta V / e_{0}$, for SiPM type MAPD-3NK0 being $6.28 \mathrm{fF}$. The quenching resistance $R_{q}$, calculated from the observed time constant $\tau \sim C_{\mu c e l l} \times R_{q}$, dropped from 5.6 M $\Omega$ by a factor of 1.4 to $4.1 \mathrm{M} \Omega$ at $100 \mathrm{~K}$.

Further characterizations of Zecotek SiPMs at liquid helium temperature are ongoing. With these measurements a deeper investigation of the influence of cryogenic temperatures on the SiPM properties was possible.

\section{Conclusion}

Several SiPM devices from Zecotek were characterized and found to be well suited for operation at cryogenic temperatures.

\section{Acknowledgments}

The authors would like to thank H. Orth from GSI, Darmstadt, for his support in obtaining the SiPMs without epoxy layer.

This material is based upon work supported by the National Science Foundation under Grant No. PHY-1309130 \& IIA-1358175 and in part by the Federal State of RhinelandPalatinate and by the Deutsche Forschungsgemeinschaft (DFG) with the Collaborative Research Center 1044.

\section{References}

[1] M. Biroth, P. Achenbach, E. Downie, A. Thomas, A low-noise and fast pre-amplifier and readout system for SiPMs, Nucl. Instrum. Methods Phys. Res. A 787 (2015) 185-188. 
90

91

[2] M. Biroth, P. Achenbach, E. Downie, A. Thomas, Silicon photomultiplier properties at cryogenic temperatures, Nucl. Instrum. Methods Phys. Res. A 787 (2015) 68-71.

[3] N. Dinu, A. Nagai, A. Para, Studies of MPPC detectors down to cryogenic temperatures, Nucl. Instrum. Methods Phys. Res. A 787 (2015) 275-279.

[4] Zecotek MAPD White Paper, Zecotek Photonics, Richmond, Canada (2011).

URL www . zecotek . com/media/MAPD-WhitePaper-March-2011.pdf 